\title{
ORIGINAL
}

\section{PREVALENCIA DE LA INFECCIÓN TUBERCULOSA Y POR EL VIH EN LOS USUARIOS DE UN PROGRAMA DE REDUCCIÓN DE RIESGOS PARA USUARIOS DE DROGAS POR VÍA PARENTERAL (UDVP)}

\author{
Miguel Álvarez. Rodríguez (1) y Pere Godoy García (2,3) \\ (1) Programa de reducción de riesgos para UDVP. Servei Obert de Lleida. \\ (2) Sección de Epidemiología. Delegación Territorial de Sanidad de Lleida. \\ (3) Facultad de Medicina Universidad de Lleida.
}

\section{RESUMEN}

Fundamento: Determinar la prevalencia de la infección tuberculosa y por el VIH, así como los factores asociados, en la población de usuarios del programa de reducción de riesgos de la ciudad de Lleida.

Métodos: La muestra la formaron los nuevos usuarios del programa en el período abril-junio de 1996, entre los los cuales se realizó un cuestionario para la recogida de datos de las variables: edad, sexo, resultado de la prueba de la tuberculina, vacunación $B C G$, conocimiento de la serologia frente al VIH, ingreso en prisión y años de consumo de heróna. Se calculó la prevalencia de la infección tuberculosa y por el VIH, con el intervalo de confianza (IC) del $95 \%$. La asociación de ambas variables con el resto de variables del estudio se determinó mediante la odds ratio (OR) y su IC del $95 \%$.

Resultados: Acudicron 150 pacientes diferentes, de los cuales 45 eran nuevos usuarios. De ellos, el $80,0 \%$ eran varones, con una edad media de 31,1 años. La prevalencia de la coinfección fue del $8,9 \%$ (IC $95 \% 2,8-22,1$ ). La prevalencia de la infección tuberculosa fue de $27,3 \%$ (IC $95 \%$ $12,4-43,0)$, siendo superior en los que tenían antecedentes de ingreso en prisión ( $\mathrm{OR}=3,4$; IC $95 \% 0,5-27,4)$. La prevalencia de la infección por el VIH fue del $36,1 \%$ (IC $95 \%$ 21,3-53,8), siendo superior en los que tenían una antigüedad. en el consumo de heroína, superior a los 11 años $(O R=7,3$; IC 95\% 1,0-65,9).

Conclusiones: El antecedente de ingreso en prisión es el principal factor de riesgo de la infección tuberculosa. Lus años de consumo se asocian con la infección por el VIH, especialmente a partir de los 11 años. Los programas de reducción de riesgos de nuestro país deberían realizar actividades de control de la infección tuberculosa y por VIH.

Palabras clave: Epidemiología. Prevalencia. Tuberculosis. $\mathrm{VIH}$

Correspondencia:

Miguel Ảlvarez Rodríguez.

Av. de Madrid, 32, 7. 2 . $^{a}$

25002 Lleida.

\section{ABSTRACT \\ Prevalence of Tuberculosis and HIV Infection Among Participants in an Intravenous Drug User Risk-Control Program}

Background: To determine the prevalence of tuberculosis and HIV infection in addition to the related factors among a population of participants in the risk control program in the town of Lleida.

Methods: The sample was comprised of the newly-enrolled participants in the program in April-June 1996, among whom a questionnaire was handed out for collecting the data concerning the variables involved: age, gender, results of the tuberculin test, BCG vaccination, knowledge of the serology regarding HIV, former imprisonment and number of years having used heroin. The prevalence of the tuberculosis and HIV infection was calculated to a $95 \%$ confidence interval $(\mathrm{Cl})$. The relating of these two variables to all other variables in the study was determined by means of the odds ratio $(O R)$ and its $95 \% \mathrm{CI}$.

Results: One hundred and fifty (150) patients were seen, 45 of whom were newly enrolled participants. Eighty percent $(80 \%)$ were inales, averaging 31.1 years of age. The prevalence of this dual infection was $8.9 \%(95 \%$ CI $2.8-22.1)$. The prevalence of the tuberculosis infection was $27.3 \%(95 \% \mathrm{CI}$ 12.4-43.0), being higher among former prison inmates $(\mathrm{OR}-3.4 ; 95 \% \mathrm{CI} 0.5-27.4)$. The prevalence of the HIV infection was $36.1 \%$ ( $95 \% \mathrm{CI} 21.3-53.8)$, being greater among those who had been using heroin for longer than 11 years $(O R=7.3$; $95 \%$ CI 1.0-65.9).

Conclusions: Former imprisonment is the main risk factor for tuberculosis infection. The number of years of heroin use are related to the HIV infection, especially when longer than 11 years. The risk control programs in our country should carry oul activities aimed at monitoring tuberculosis and HIV infection.

Key words: Epidemiology. Prevalence. Tuberculosis. HIV. 


\section{INTRODUCCIÓN}

Dentro de las diferentes estrategias llevadas a cabo desde la administración para la asistencia a toxicómanos y, en concreto, a los usuarios de drogas por vía parenteral (UDVP), se pusieron en marcha los programas de reducción de riesgos asociados al consumo de sustancias por esta vía ${ }^{1.2}$.

Uno de los objetivos de estos programas es contactar con una serie de usuarios de drogas que por su situación de consumo activo, no están relacionados con ningún centro asistencial. Por este motivo, este tipo de programas, se centran más en el "cómo» se consume que en el «qué» se consume ${ }^{3}$.

Es precisamente por este "cómo» se consume, que enfermedades como la hepatitis $\mathrm{B}$, la Hepatitis $\mathrm{C}$ y el sida adquieren una gran importancia en este colectivo. Por otro lado, la condición de ilegalidad de las sustancias consumidas, favorece la entrada en circuitos de marginación que, a su vez, facilitan la aparición de otras enfermedades, como la tuberculosis, que no están directamente vinculadas con la utilización de la vía parenteral pero sí, con las condiciones de vida de estas personas ${ }^{4-8}$.

El Estado español presenta una de las tasas más altas de sida y tuberculosis de Europa, y ambas epidemias están ampliamente solapadas en nuestro medio. Ello tiene especial relevancia debido a que el riesgo de presentar tuberculosis en las personas coinfectadas por el virus de la inmunodeficiencia humana (VIH), es muy superior al de la población general.

También, en la ciudad de Lleida, se ha constatado la influencia negativa del VIH en la evolución de las tasas de incidencia por tuberculosis. Así, en el período 1992-1996, la proporción de nuevos casos de tuberculosis coinfectados por el VIH fue del $24 \%{ }^{9}$. En esta ciudad se puso en marcha un programa de reducción de riesgos para UDVP en diciembre de 1993. Dentro de las diferentes actividades que se llevaron a cabo, se inició el presente estudio, con el objetivo de determinar la prevalencia de la infección por el VIH y la infección tuberculosa entre los nuevos usuarios del programa, así como determinar posibles factores de riesgo.

\section{MATERIAL Y MÉTODOS}

La población del estudio eran los usuarios del único programa de reducción de riesgos para UDVP que existe en la ciudad de Lleida. Este municipio tiene censada una población de 112.093 habitantes y presenta las características propias de un municipio urbano de tamaño medio, con un importante sector de servicios y un nivel medio-bajo de industrialización. Este programa de reducción de riesgos se basa fundamentalmente en el intercambio de jeringuillas, pero mantiene una estrecha coordinación con el Centro de Alcoholismo y otras Toxicomanías, donde se lleva a cabo un programa de mantenimiento con metadona.

La muestra del estudio estuvo constituida por los nuevos usuarios que acudieron al programa, en el período comprendido entre abril y junio de 1996. Se elaboró un cuestionario, para la realización del presente trabajo, a través del cual se recogieron los datos de las variables a estudio mediante entrevista personal con cada usuario.

Las variables del estudio fueron la edad, el sexo, el resultado de la prueba de la tuberculina (en mm), vacunación BCG (confirmación mediante la visualización de la cicatriz), conocimiento de la serología frente al VIH (se consideró positiva la respuesta afirmativa y negativa o desconocida las personas que conocían su seronegatividad o no se habían realizado la prueba), antecedente de ingreso en prisión, ejercicio de la prostitución y años de consumo de heroína.

La prueba de la tuberculina se realizó con 2 unidades de PPD RT 23, mediante inyección intradérmica según técnica de Mantoux. La lectura del resultado se efectuó entre las 48-72 horas posteriores, a través de la delimitación 
de la induración mediante palpación suave con el dedo. Se consideraron positivas las induraciones de 5 o más milímetros en no vacunados con BCG y 15 milímetros en los vacunados ${ }^{10}$. También se consideraron positivos los casos con constancia documentada de haber prescntado una tuberculosis activa.

Se calculó la prevalencia de la infección tuberculosa, de VIH y para ambas infecciones simultáneamente, con su intervalo de confianza del 95\%. La asociación de riesgo de infección tuberculosa, por el VIH o la coinfección con el resto de variables del estudio se determinó con la odds ratio (OR) y su intervalo de confianza del 95\% (IC 95\%). La asociación de riesgo de infección por VIH con los años de consumo de heroína se estudió mediante la prueba de $\chi^{2}$ de tendencia para determinar la existencia de dosis respuesta para tres niveles de cxposición: menos de 8 años de consumo, de 8 a 11 años y más de 11 años. Los datos se analizaron con el programa Epi Info 6.04.

\section{RESULTADOS}

En el período del estudio acudieron al programa de reducción de riesgos 150 sujetos diferentes, de los cuales 45 eran nuevos usuarios del programa. El número total de personas contactadas fue de 2.481 en el citado período. De los 45 participantes en el estudio, el $80,0 \%$ eran varones, con una edad media de 31,1 años (desviación estandar -DE - de 5,3), y un 60,0\% tenían antecedentes de ingreso en algún centro penitenciario. La prevalencia de la infección tuberculosa fue del $27,3 \%$ (IC 95\% 12,4-43,0) (tabla 1). En 2 casos, se consideró la prueba positiva, dado que tenían antecedentes de haber presentado tuberculosis activa. La prevalencia fue superior en las mujeres $(33,3 \%)(\mathrm{OR}=1,4 ; \mathrm{IC} 95 \% 0,2-8,9)$ y en $\operatorname{los}$ mayores de 30 años $(36,0 \%) \quad(\mathrm{OR}=3,0$; IC95\% 0,6-17,6). Así mismo, la infección por el bacilo de Koch, fue superior en los pacientes que tenían antecedente de ingreso en prisión $(\mathrm{OR}=3,4$; IC95\% 0,5-27,4) y an- tecedente de vacunación $\mathrm{BCG}(\mathrm{OR}=2,6$; IC95\% 0,5-12,7) pero las diferencias no fueron estadísticamente significativas (tabla 2). El tamaño de la reacción tuberculínica presentó una media de 21,9 mm (DE: 7,7). Excepto una prueba de $12 \mathrm{~mm}$ en un no va-

Tabla 1

Prevalencia de la infección tuberculosa (Tbc) y por el VIH

\begin{tabular}{|lcr|}
\hline & Prevalencia (\%) & IC 95\% \\
\hline Infccción Tbc & 27,3 & $12,4-43,0$ \\
Infección VIH & 36,3 & $21,3-53,8$ \\
Coinfección VIH/Tbc & 8,9 & $2,8-22,1$ \\
\hline
\end{tabular}

Tabla 2

Factores relacionados con la infección tuberculosa

\begin{tabular}{|c|c|c|c|c|}
\hline \multirow{2}{*}{ Factor } & \multicolumn{2}{|c|}{ Infección } & \multirow{2}{*}{$O R$} & \multirow{2}{*}{ IC $95 \%$} \\
\hline & $n / N$ & $\%$ & & \\
\hline \multicolumn{5}{|l|}{ Edad } \\
\hline$<30$ & $3 / 19$ & 15,8 & & \\
\hline $30+$ & $9 / 25$ & 36,0 & 3,0 & $0,6-17,6$ \\
\hline \multicolumn{5}{|l|}{ Sexo } \\
\hline Hombre & $9 / 35$ & 25,7 & & \\
\hline Mujer & $3 / 9$ & 33,3 & 1,4 & $0,2-8,9$ \\
\hline \multicolumn{5}{|l|}{$\mathrm{BCG}$} \\
\hline Sí & $6 / 15$ & 40,0 & 2,6 & $0,5-12,7$ \\
\hline No & $6 / 29$ & 20,7 & & \\
\hline \multicolumn{5}{|c|}{ Años de consumo } \\
\hline$<10$ & $6 / 20$ & 30,0 & & \\
\hline 10 o más & $3 / 14$ & 21,4 & 0,6 & $0,1-4,0$ \\
\hline \multicolumn{5}{|l|}{ Prisión } \\
\hline Sí & $10 / 29$ & 35,4 & 3,4 & $0,5-27,4$ \\
\hline No & $2 / 15$ & 13,3 & & \\
\hline \multicolumn{5}{|l|}{ Ac HIV } \\
\hline Sí & $4 / 13$ & 30,8 & & \\
\hline No & $6 / 13$ & 46,1 & 1,3 & $0,2-7,2$ \\
\hline \multicolumn{5}{|l|}{ Prostitución } \\
\hline Sí & $3 / 6$ & 50,0 & - & - \\
\hline No & $0 / 3$ & 0,0 & & \\
\hline
\end{tabular}

OR: odds ratio; IC: Intervalo de confianza. 
cunado con BCG, el resto, presentó induraciones iguales o superiores a $15 \mathrm{~mm}$.

La prevalencia de la infección por el VIH fue del 36,1\% (IC 95\% 21,3-53,8) siendo superior en las mujeres $(55,5 \%)$. Así mismo, la infección por VIH fue superior en los UDVP que tenían antecedentes de ingreso en prisión ( $\mathrm{OR}=1,2$; IC95\% 0,2-6,6), pero las diferencias tampoco fueron estadísticamente significativas (tabla 3 ).

Tabla 3

Factores relacionados con la infección por el VIH

\begin{tabular}{|c|c|c|c|c|}
\hline \multirow{2}{*}{ Factor } & \multicolumn{2}{|c|}{ Infección VIH } & \multirow{2}{*}{$O R$} & \multirow{2}{*}{ IC $95 \%$} \\
\hline & $n / N$ & $\%$ & & \\
\hline \multicolumn{5}{|l|}{ Edad } \\
\hline$<30$ & $5 / 14$ & 35,7 & 1,0 & $0,1-4,5$ \\
\hline $30+$ & $8 / 22$ & 36,3 & & \\
\hline \multicolumn{5}{|l|}{ Sexo } \\
\hline Hombre & $8 / 27$ & 29,6 & & \\
\hline Mujer & $5 / 9$ & 55,5 & 3,0 & $0,5-19,0$ \\
\hline \multicolumn{5}{|l|}{ Prostitución } \\
\hline Sí & $4 / 6$ & 66,6 & 4,0 & $0,1-252,4$ \\
\hline No & $1 / 3$ & 33,3 & & \\
\hline \multicolumn{5}{|l|}{ Prisión } \\
\hline Sí & $9 / 24$ & 37,5 & 1,2 & $0,2-6,7$ \\
\hline No & $4 / 12$ & 33,4 & & \\
\hline \multicolumn{5}{|c|}{ Infección Tbc } \\
\hline Sí & $4 / 10$ & 40,0 & 1,3 & $0,2-7,7$ \\
\hline No & $9 / 26$ & 34,6 & & \\
\hline
\end{tabular}

OR: odds ratio; IC: Intervalo de confianza.

La coinfección por el Mycobacterium tuberculosis y por el VIH tuvo una prevalencia de 8,9\% (IC 95\% 2,8-22,1). El riesgo de coinfección aumentó con los años de consumo. Así, la prevalencia en los que presentaron un antecedente de consumo inferior a 8 años fue de $21,4 \%$, para aquellos con un consumo entre 8 y 11 años fue de $40,0 \%$, y para los que tenían un antecedente superior a los 11 años del 66,7\% (tabla 4). La prueba de $\chi^{2}$ de tendencia resultó estadísticamente significativa $(\mathrm{p}=0,02)$.

\section{Tabla 4}

Asociación de los años de consumo con la infección por el VIH

\begin{tabular}{|c|c|c|c|c|c|c|}
\hline \multirow{3}{*}{$\begin{array}{l}\text { Años de } \\
\text { consumo }\end{array}$} & \multicolumn{4}{|c|}{ Infección VIH } & \multirow{3}{*}{$O R$} & \multirow{3}{*}{ IC $95 \%$} \\
\hline & \multicolumn{2}{|r|}{$S i$} & \multicolumn{2}{|c|}{ No } & & \\
\hline & $n$ & $\%$ & $n$ & $\%$ & & \\
\hline$<8$ años & 3 & 21,4 & 11 & 78,6 & 1,0 & \\
\hline $8-11$ & 2 & 40,0 & 3 & 60,0 & 2,4 & $0,2-37,9$ \\
\hline$>11$ & 8 & 66,7 & 4 & 33,3 & 7,3 & $1,0-65,9$ \\
\hline
\end{tabular}

OR: odds ratio; IC: intervalo de confianza.

\section{DISCUSIÓN}

El estudio ha permitido caracterizar a la población que contacta con un programa de reducción de riesgos para UDVP, en un municipio urbano de tamaño medio $\mathrm{y}$ ha puesto de manifiesto, al igual que otros estu$\operatorname{dios}^{12,13}$, la alta prevalencia de la coinfección por Mycobacterium tuberculosis y VIH en este colectivo. Al mismo tiempo que evidencia la elevada prevalencia de ambas infecciones por separado, superiores a las existentes para el grupo de edad en el conjunto de la población española ${ }^{14}$.

Hay que tener en cuenta que, en el caso de la infección tuberculosa, tanto la vacunación $\mathrm{BCG}$, como la infección por VIH podrían alterar los resultados de la prueba de la tuberculina. La vacuna BCG podría ser responsable de algunas reacciones a la tuberculina, dando lugar a falsos resultados positivos que habrían inducido a clasificar erróneamente como infectado a algún individuo vacunado. Sin embargo, dado que las reacciones atribuibles al BCG se suelen situar por debajo de $15 \mathrm{~mm}$, que la media de las reacciones en nuestro estudio fue de 21,9 $\mathrm{mm}$, y que todas las reacciones, excepto una, presentan induraciones superiores a 15 $\mathrm{mm}$, es poco probable que el BCG haya interferido los resultados ${ }^{11}$. 
Asimismo, en el caso del VIH la disminución de la inmunidad induciría la posible presentación de falsos negativos a la prueba de tuberculina que se asociarían con una infraestimación de la prevalencia de la infección. Por tanto, cabe pensar que la prevalencia de infección tuberculosa del $27,3 \%$ sería la prevalencia mínima de este colectivo.

Respecto a la infección por VIH, la prevalencia $(36,1 \%)$, está basada en el conocimiento de los resultados de la serología por parte de los sujetos, por tanto es posible que haya una subestimación de la misma, tanto por desconocimiento de la serología, como por la ocultación de la seropositividad. Sin embargo, otros estudios han demostrado una aceptable validez de la autonotificación del estado serológico ${ }^{15}$. Esta prevalencia autonotificada tiende a disminuir en los últimos años, según muestran otros estur $\operatorname{dios}^{16,17}$. En esta progresiva disminución ha influido, de manera notable, los nuevos hábitos en el consumo de heroína (vía de administración inhalada), propiciados por el llamado «miedo al sida» entre los consumidores de sustancias ilegales y, por otro lado, el progresivo incremento en nuestro medio de heroína de tipo "marrón» (brown sugar), más eficaz por vía inhalada ${ }^{1,4}$.

Se constata en el estudio que, cuanto más tiempo se lleva consumiendo, mayor es la prevalencia de infección por el VIH, con una prevalencia superior al $40,0 \%$ a partir de los 8 años de consumo. Sin duda, ello está relacionado con las mayores posibilidades de realizar conductas de riesgo cuanto mayor es el tiempo de permanencia en la subcultura de las drogas, tal como han demostrado otros estudios ${ }^{18-20}$. A parte de la vía de administración de sustancias tóxicas, hay que tener en cuenta que en el colectivo de UDVP, las relaciones sexuales son una moneda de cambio habitual a la hora de financiarse la adicción. La prevalencia superior de la infección por el VIH observada en las mujeres, en este y otros estudios, podría relacionarse con esta vía de transmisión. En este sentido, no sólo hay que pensar en la prostitución per se, sino en las dificultades de planificar (o negociar) un sexo más seguro, tanto bajo los efectos de estas sustancias, como en situaciones de síndrome de abstinencia ${ }^{21-23}$.

Por lo tanto, tendríamos las dos vías de transmisión del VIH más frecuentes en nuestro medio (compartir material de inyección y relaciones sexuales sin protección), unidas en un colectivo en el que la primera medida de prevención debiera ser poner a disposición los elementos necesarios para la misma, es decir, material de inyección estéril, programas de mantenimiento con metadona de bajo umbral y preservativos, todo ello con un acceso fácil $\mathrm{y}$, evidentemente, sin perjuicio de la existencia de otros programas con objetivos de mayor exigencia $^{1-3,14,24-26}$.

Respecto a la coinfección tuberculosis-VIH, la prevalencia $(8,9 \%)$ cs muy elcvada en el colectivo estudiado y pone de manifiesto no sólo que en nuestra ciudad ambas epidemias estan ampliamente solapadas (tal como se ha puesto repetidamente de manifiesto por otros estudios en nuestro país), sino la importancia de factores de riesgo tales como el hábito de compartir el material de inyección, entre los UDVP, y el antecedente de ingreso en prisión ${ }^{17,21,25,26}$. No debemos olvidar que el ser UDVP, ya era un factor de riesgo para la infección por M. tuberculosis antes de la era del sida, por lo tanto, la aparición del mismo no ha hecho más que agravar una situación conocida ${ }^{14}$. Y no, únicamente, por cuestiones de tipo sanitario (posibilidad de reactivación de tuberculosis endógenas), sino por temas de carácter social, ya que el progresivo deterioro en el que se ven inmersos estas personas favorece, aún más si cabe, su marginación social y dificulta el acceso a los recursos convencionales.

Por lo tanto, la necesidad de instaurar programas específicos de prevención y control de estas infecciones, en todos los recursos comunitarios (centros de acogida, pro- 
gramas de mantenimiento con metadona, centros penitenciarios, programas de tratamiento) que entran en contacto con este tipo de población, es evidente ${ }^{14,26}$. Sin embargo no debemos olvidar que, al mismo tiempo que instauramos programas preventivos, debemos crear las infraestructuras necesarias de acercamiento a estas personas. Más aún, si tenemos en cuenta que la adicción es una enfermedad crónica con recaídas y que cada vez serán poblaciones de más difícil acceso para los recursos convencionales.

\section{BIBLIOGRAFÍA}

1. O'Hare PA. Apuntes sobre el concepto de reducción de daños. En: O'Hare PA, Newcombe R, Matthews A, Buning EC, Drucker E. La reducción de los daños relacionados con las drogas (edición española). Barcelona: Grup IGIA; 1995; p. 17-39.

2. Ministerio de Sanidad y Consumo. Guía para la puesta en marcha de programas de intercambio de jeringuillas. Madrid: Ministerio de Sanidad y Consumo; 1996.

3. Plan Nacional sobre Drogas. Centros de encuentro y acogida. Documento de referencia. Barcelona: Grup Igia; 1995.

4. Baulurz G, Kaminski D, Zambek S. Le Bon Usage. Prévention du SIDA à l'adresse des usagers de drogues. Bruxelles: Agence Prévention SIDA; 1992.

5. Vidal Pla R, de Gracia Roldán X. Tratamiento de la tuberculosis en la infección por VIH. En: Vidal Pla R, de March Ayuela P. Monografias Clínicas en Neumologia. Barcelona: Ediciones Doyma S. A; 1992; p. 93-101.

6. Marlatt GA. Reducción de daños: Tal como es. Addictive Behaviors. 1996; 21: 779-788.

7. Caylà JA, Jansà JM, Serrano J, Garrell E. Evolución por el VIH y programas con adecuada capacidad de retención en el control del SIDA y la tuberculosis en adictos a drogas por vía parenteral. Med Clin (Barc) 1992; 98: 636.

8. Friedman Lloyd N, Williams Michael T, Singh Tcjinder P, Frieden Thomas R. Tuberculosis AIDS, and death among substance abusers on welfare in New York City. N Engl J Med 1996; 334: 828-833.
9. Godoy P, Artigues A, Torres J, Alsedà M, Mirada G, Bach P. Factores asociados con los casos de tuberculosis coinfectados por el virus de la inmunodeficiencia humana. Gac Sanit 1997; 11 (Supl 1): 51 .

10. Grupo de trabajo sobre tuberculosis. Fondo de investigación sanitaria (FIS). Consenso nacional para el control de la tuberculosis en España. Med Clin (Barc) 1992; 98: 24-31.

11. Grupo de Estudios de Contactos de la Unidad de Investigación en Tuberculosis de Barcelona (UITB) Documento de consenso sobre el estudio de contactos en los pacientes tuberculosos. Med Clín (Barc) 1999; 112: 151-156.

12. Solera J, López E, Serna E, Vergara L, Martínez-Alfaro F, Sáez. I. Riesgo de tuberculosis en adictos a drogas por vía parenteral seropositivos frente al virus de la inmunodeficiencia humana. Un estudio de cohortes en comunidades para deshabituación de toxicómanos. Med Clín (Barc) 1993; 100: 725-729.

13. Martín Sánchez V, Álvarez Guisasola F, Caylà J, Álvare 2 JL. Predictive Factors of Mycobacterium tuberculosis Infection and Pulmonary Tuberculosis in Prisioners. Int J Epidemiol 1995; 24: 630-636.

14. Rey R, Ausina V, Casal M, Caylà J, De March P, Moreno $S$ et al. Situación actual de la tuberculosis en España. Una perspectiva sanitaria en precario con respecto a los países desarrolados. Med Clin (Barc) 1995; 105: 703-707.

15. Mc Cuskerr J, Stoddard AM, McCarthy E. The validity of self-reported HIV antibody test results. Am J Public Health 1992; 82: 567-569.

16. Alba A, Terán A, Cuende JI, Sánchez S, González C. Prevalencia de la infección por el virus de la inmunodeficiencia humana en drogodependientes atendidos desde 1991 a 1996 en un centro de desintoxicación de Castilla y León. Enferm Infecc Microbiol Clin 1998; 16: 169-174.

17. Pérez-Aguado F, Alonso Moreno FJ, Urbina Torija J. Prevalencia de infección por el virus de la inmunodeficiencia humana tipo 1 y de Mycobacterium tuberculosis en una población reclusa entre los años 1989-1995. Med Clín (Barc) 1998; 110: 167-170.

18. Rodés A, Vall M, Casabona J, Nuez M, Rabella N, Mitrani L. Prevalencia de la infección por el virus de la inmunodeficiencia humana y de los comportamientos asociados a su transmisión entre usuarios de drogas por vía parenteral seleccionados en la ca1le. Med Clin (Barc) 1998; 111: 372-377.

19. Zunzunegui MV, Rodríguez MA, Sarasqueta C. Drogadicción intravenosa y riesgo de infección por el VIH en Madrid 1990. Gac Sanit 1993; 7: 2-11. 
20. Bravo MJ, Barrio G, De la Fuente L, Colomo C, Royuela L, Estébanez P. Conductas de riesgo para la transmisión del VIH entre usuarios recientes de un programa de intercambio de jeringas en Madrid, 1993. Gac Sanit 1996; 10: 261-273.

21. van Ameijden EJC, van der Hoek JAR, van Haastrecht HJA, Coutinho RA. The Harm Reduction Approach and Risk Factors for Human Immunodeficiency Virus (HIV) Seroconversion in Injecting Drug Users, Amsterdam. Am J Epidemiol 1992; 36: 236-342.

22. Pérez González K, Domingo-Salvany A, Hartnoll R. Prevalencia de la infección por el virus de la inmunodeficiencia humana y conductas de riesgo en consumidores de opioides visitados en un servicio de urgencias. Gac Sanit 1999; 13: 7-15.

23. Alfonso Gil R, Hurtado Navarro I, Espacio Casanovas A, Santos Rubio C, Tomás Dols S. Prácti- cas de riesgo y seroprevalencia al VIH, VHB y VHC en los pacientes del Centro de Información y Prevención del SIDA de Valencia. Gac Sanit 1999; 13: 16-21.

24. De la Fuente L. Control de los problemas de salud asociados al consumo de drogas en España: hacia un abordaje científico y priorizado. Gac Sanit 1996; 10: 255-260.

25. Martín V, Caylà, Bolea A, de Paz JA. Evolución de la prevalencia de la infección por Mycobacterium tuberculosis en una población reclusa al ingreso en prisión entre 1991 y 1996. Med Clín (Barc) 1998; 111: 11-16.

26. Godoy P, Castilla J, Rullán JV. Incidencia y factores de riesgo de la asociación del SIDA y la tuberculosis en Fspaña. Med Clin (Barc) 1998; 110: 205-208 